\title{
Gene Polymorphisms and Circulating Levels of MMP-2 and MMP-9: A Review of Their Role in Breast Cancer Risk
}

\author{
SUÉLÈNE GEORGINA DOFARA ${ }^{1,2,3}$, SUE-LING CHANG ${ }^{1,2}$ and CAROLINE DIORIO ${ }^{1,2,3}$ \\ ${ }^{1}$ Division of Oncology, CHU de Québec-Université Laval Research Center, Quebec City, QC, Canada; \\ ${ }^{2}$ Cancer Research Center - Laval University, Quebec City, QC, Canada; \\ ${ }^{3}$ Department of Social and Preventive Medicine, Faculty of Medicine, Laval University, Quebec City, QC, Canada
}

\begin{abstract}
MMP-2 and MMP-9 genes have been suggested to play a role in breast cancer. Their functions have been associated with invasion and metastasis of breast cancer; however, their involvement in the development of the disease is not well-established. Herein, we reviewed the literature investigating the association between circulating levels and polymorphisms of MMP-2 and MMP-9 and breast cancer risk. Various studies report conflicting results regarding the relationship of polymorphisms in MMP-2 and MMP-9 and breast cancer risk. Nevertheless, it appears that the T allele in rs243865 and rs2285053 in MMP-2 are associated with reduced risk of breast cancer. In addition, high levels of latent form and low levels of active form of MMP-2 were observed in breast cancer patients compared to controls. For MMP-9, high latent levels and low total levels were found in breast cancer patients compared to controls. Additional studies are needed to comprehend the role of these genes in breast carcinogenesis.
\end{abstract}

Matrix metalloproteinases (MMP) are a family of $\mathrm{Zn}^{2+}$ dependent endopeptidases responsible for cleaving components of the extracellular matrix (1). They are classified into several families according to their structural differences $(1,2)$. MMP-2 and -9 comprise the gelatinase family that possesses three fibronectin repeats allowing for degradation of denatured collagen (gelatin) and collagens IV and V $(2,3)$. These gelatinases degrade collagen in the basement membrane

This article is freely accessible online.

Correspondence to: Caroline Diorio, CHU de Québec-Université Laval Research Center (Oncology Division), 1050 Chemin SainteFoy, Quebec City, QC, G1S 4L8, Canada. Tel: +1 4186827390, Fax: +1 4186827949, e-mail: caroline.diorio@crchudequebec.ulaval.ca

Key Words: Matrix metalloproteinases, gelatinases, polymorphisms, circulating levels, breast cancer risk, review.
(4), as well as other extracellular matrix components, thus promoting extracellular matrix remodeling and consequently play a key role in several physiological processes, such as tissue repair, wound healing, and cell differentiation $(5,6)$.

Gelatinases could be involved in carcinogenesis processes, including cell proliferation, angiogenesis, and tumor metastasis through their proteolytic function (7). Indeed, the literature suggests their involvement in several pathological processes critical for cancer development, including inflammation, angiogenesis, and cell proliferation, as well as in tumor progression $(8,9)$. More specifically, the biological functions of MMP-2 and -9 proteins have been associated with invasive and metastatic stages of breast cancer $(10,11)$; however, their involvement in breast cancer development is unclear.

Herein, we aimed to review and discuss articles which studied the association between gelatinases and breast cancer risk. For this purpose, an electronic search of the MEDLINE (PubMed) database was performed to identify all published studies that evaluated the association of polymorphisms or circulating levels of MMP-2 and MMP-9 with breast cancer risk.

\section{Matrix Metalloproteinase-2 (MMP-2)}

$M M P-2$ is located on chromosome 16 and codes for gelatinase A. The substrates for this enzyme include gelatin, collagen $\mathrm{V}$, and collagen VI (12). The $M M P-2$ gene has been studied in several abnormal physiological processes, such as obesity and cancer (13). Polymorphisms that alter the function and efficacy of this protein could be associated with breast cancer risk. Several studies have evaluated this association (14-23).

\section{Circulating Levels of MMP-2 and Breast Cancer Risk}

MMP-2 exists in three forms: latent, active and total. These forms vary in their molecular weight, making it possible to measure them in the blood. Enzymatic techniques such as 
zymography $(12,24)$ allow quantifying the presence of these different MMP-2 forms in the blood. However, there are other less specific methods to measure circulating levels. Studies that have examined circulating levels of MMP-2 and breast cancer risk have measured either the latent, active or concentrations of both combined (latent plus active) (25-30), but two studies did not specify the form $(31,32)$. Eight research articles on circulating levels of MMP-2 are presented in Table I.

Latent form (pro-MMP-2). MMP-2 is expressed in its latent form and is activated in the extracellular matrix. This latent form can be measured in either serum or plasma. The molecular weight of pro-MMP-2 is $72 \mathrm{kDa}$ (33). La Rocca et al. (25) found that the serum levels of the pro-MMP-2 form were higher in breast cancer patients than in healthy patients $(p<0.0001)$. The authors used the zymography technique to quantify the enzyme in the study.

Active MMP-2 form (aMMP-2). Active MMP-2 results from the activation of the pro-MMP-2 form by proteolytic cleavage of the N-terminus (34). The molecular weight of this active species is $63 \mathrm{kDa}$ (33). This functional form of MMP-2, as measured in plasma, was reportedly lower in breast cancer patients than in healthy people $(100 \mathrm{pg} / \mathrm{mg} v s$. $130 \mathrm{pg} / \mathrm{mg}, p=0.038$ ) (26). The concentrations of aMMP-2 in serum were also lower in breast cancer patients than in healthy groups (375 pg/mg vs. $725 \mathrm{pg} / \mathrm{mg}, p<0.001)$ (27). Thus, circulating levels of aMMP-2 appear to be lower in breast cancer patients than healthy people.

Total MMP-2 (tMMP-2). This form of MMP-2 includes both the pro-enzyme and the active enzyme. Somiari et al. (26) found that the plasma concentrations of tMMP-2 were higher in patients with breast cancer than healthy individuals ( $1350 \mathrm{pg} / \mathrm{mg} v s .900 \mathrm{pg} / \mathrm{mg}, p=0.002$ ). However, Aroner et al. (30) and Kim et al. (29) did not find any association between plasma levels of tMMP-2 in breast cancer cases and healthy individuals. In addition, two studies revealed that tMMP-2 concentrations, when assessed in the serum, did not differ between breast cancer patients and healthy low risk women $(p=0.926)$ (27) or healthy women $(p>0.05)$ (28). Thus, tMMP-2 does not appear to be associated with breast cancer. These results for tMMP-2 levels are not surprising since opposite associations have been observed for pro-MMP-2 and aMMP-2 levels with the risk of breast cancer.

No specific form. Other authors have studied circulating levels of MMP-2 in breast cancer without identifying the form. Two studies found that serum mean levels of MMP-2 are higher in patients with breast cancer than in healthy patients $(694.3 \mathrm{ng} / \mathrm{ml}$ vs. $593.3 \mathrm{ng} / \mathrm{ml}$ (31), $806.5 \mathrm{ng} / \mathrm{ml} v s$.
$771.2 \mathrm{ng} / \mathrm{ml}(32), p<0.05)$. These results are nonetheless concordant with those observed between pro-MMP-2 levels and breast cancer risk.

\section{MMP-2 Gene Polymorphisms and Breast Cancer Risk}

A total of 37 polymorphisms located on the $M M P-2$ gene were studied, with most located in the promoter region. The main studied polymorphisms were rs243865, rs2285053, rs243866 and rs243864. Studies of $M M P-2$ polymorphisms are described in Table II.

$r s 243865$. The rs243865 polymorphism in $M M P-2$ is a common $\mathrm{C} \rightarrow \mathrm{T}$ transition at position -1306 in the promoter. This transition interrupts binding with stimulating protein 1 (Sp1), which is a transcription factor. It has been reported that the $\mathrm{T}$ allele reduces the expression of MMP-2 (35). Ten studies investigated the association between rs243865 and breast cancer risk, but the results were unclear (14-23). Several authors have hypothesized that this polymorphism may reduce the risk of breast cancer because of less protein expression. Three studies found a significant association between rs243865 in $M M P-2$ and risk of breast cancer in Chinese $(\mathrm{OR}=0.46 ; 95 \% \mathrm{CI}=0.34-0.63 ; p=0.00001)$ (14), Mexican $(\mathrm{OR}=0.47 ; 95 \% \mathrm{CI}=0.24-0.88 ; p=0.01)(17)$ and Tunisian $(\mathrm{OR}=0.39 ; 95 \% \mathrm{CI}=0.25-0.72)$ (22) populations. These three studies used the dominant model and found that $\mathrm{CT}+\mathrm{TT}$ genotypes reduced the risk of breast cancer compared to the CC genotype. However, Saeed et al. (19) also used this same dominant model in the Saudi population and found that $\mathrm{CT}+\mathrm{TT}$ genotypes increased the risk of breast cancer compared to the $\mathrm{CC}$ genotype $(\mathrm{OR}=2.12 ; 95 \%$ $\mathrm{CI}=1.09-4.11 ; p=0.025)$. It is important to note that, deviation from Hardy-Weinberg equilibrium was observed for rs243865 in this study (19). Using a recessive model in a Caucasian-Hispanic population, Slattery et al. (21) observed that the association between TT carriers compared to $\mathrm{CT}+\mathrm{CC}$ carriers was significant $(\mathrm{OR}=0.84$; $95 \% \mathrm{CI}=0.73$ 0.97 ) after adjustment for body mass index and other risk factors. Additionally, five studies analyzed the relation between rs243865 in $M M P-2$ and breast cancer risk in Caucasian (15, 20), Brazilian (16), Chinese (18) and Tunisian (23) populations, but did not find any association. In contrast with studies reporting decreased breast cancer risk with rs243865, these four former studies used an additive model. Increasing the number of copies of the $\mathrm{T}$ allele did not affect the risk of breast cancer. Habel et al. (23) compared the $\mathrm{T}$ allele to the $\mathrm{C}$ allele of rs243865 in $M M P-2$ in the Tunisian population and demonstrated that the $\mathrm{T}$ allele was not associated with the risk of breast cancer. Taken together, we cannot exclude the possibility that the $\mathrm{T}$ allele may reduce breast cancer risk in some populations. 
rs243866. rs243866 is a $\mathrm{G}$ to A transition located in the $M M P-2$ promoter, at position -1575. MMP-2 is estrogenresponsive, but the $-1575 \mathrm{G} \rightarrow \mathrm{A}$ transition appears to be an incomplete palindromic binding site for estrogen receptor and the $-1575 \mathrm{~A}$ allele reduces the transcriptional activity of MMP-2 (36). According to two studies in Chinese (18) and Tunisian (23) populations, rs243866 was not associated with breast cancer risk $(p>0.05)$. These results are consistent with the fact that the $G$ and $A$ alleles have similar allelic expression. The A allele is likely non-functional (35).

$r s 243864$. This polymorphism is located in the $M M P-2$ promoter at position -790 and involves a transition of the common allele $\mathrm{G}$ to $\mathrm{T}$. The functional significance of the wild $\mathrm{T}$ allele is unclear. The rs 243864 polymorphism has been studied in Chinese (18) and Tunisian (23) populations, but none of these studies found an association between this polymorphism and breast cancer risk $(p>0.05)$.

$r s 2285053$. rs 2285053 is located in the MMP-2 promoter at position -735 and implicates a transition of the common allele $\mathrm{C}$ to T. To our knowledge, the biological significance of the wild $\mathrm{T}$ allele is undefined. However, Yu et al. (37) have reported that according to bioinformatics analyses, rs2285053 in $M M P-2$ could alter a Sp1 binding site and influence $M M P$ 2 transcription. Three studies were identified that investigated the association between rs2285053 in $M M P-2$ and breast cancer risk. Two studies showed that the rs2285053 T allele rather than the $\mathrm{C}$ allele reduced the risk of breast cancer in the Tunisian $(\mathrm{OR}=0.59 ; 95 \% \mathrm{CI}=0.46-0.75)$ (23) and Iranian $(\mathrm{OR}=0.61 ; 95 \% \mathrm{CI}=0.37-0.99 ; p=0.049)$ (38) populations. However, Beeghly-Fadiel et al. (18) did not find any association between CC, CT, TT genotypes of rs2285053 and breast cancer risk in additive models $(p=0.436)$. Based on this evidence, the $\mathrm{T}$ allele may affect breast cancer risk.

Other polymorphisms in MMP-2. Thirty-three other polymorphisms in $M M P-2$ were also examined in two studies $(18,21)$. However, none of these polymorphisms was associated with breast cancer risk in the study populations, except for rs 11541998 that CG+GG increased breast cancer risk compared to $\mathrm{CC}(\mathrm{OR}=1.16 ; 95 \% \mathrm{CI}=1.02-1.31)(21)$.

Haplotypes in MMP-2. Haplotype analyses have also been performed. Beeghly-Fadiel et al. (18) observed significant haplotype effects of rs11644561 and rs11643630 on breast cancer risk. The authors found that the haplotype with minor alleles (AG) for both SNPs was associated with reduced breast cancer risk $(\mathrm{OR}=0.6 ; 95 \% \mathrm{CI}=0.4-0.8 ; p=0.003)$ compared to the haplotype with both major alleles (GT). In the same study, no significant haplotype effects for rs243865 and rs2285053 were observed. However, Habel et al. (23) found that patients who had GCTT and GTTC combinations of rs243866, rs243865, rs243864 and rs2285053 respectively had a lower risk of breast cancer (GCTT: OR $=0.49,95 \%$ $\mathrm{CI}=0.25-0.94$; GTTC: $\mathrm{OR}=0.39,95 \% \mathrm{CI}=0.19-0.81)$ than those who had GCTC haplotypes. Several studies are needed to clarify the haplotype effect of polymorphisms located in $M M P$-2 gene on breast cancer risk.

\section{Matrix Metalloproteinase-9 (MMP-9)}

The $M M P-9$ gene is located on chromosome 20 and encodes the gelatinase B protein. MMP-9 expression is either lower or absent in normal tissues, and elevated in inflammation and wound healing (39). The main substrates for this enzyme include gelatin, collagen IV, and V (12).

\section{Circulating Levels of MMP-9 and Breast Cancer Risk}

Few studies have evaluated the role of circulating levels of MMP-9 in carcinogenesis. Similar to gelatinase A, gelatinase $\mathrm{B}$ is also translated into a pro-enzymatic form and activated in the extracellular space. Six studies of circulating levels of MMP-9 are presented in Table I.

Pro-MMP-9. This form is the latent form of MMP-9 and has a molecular weight of $92 \mathrm{kDa}$ (33). In a study conducted by La Rocca et al., the serum concentrations of pro-MMP-9 were significantly higher in women with breast cancer than in healthy women $(p<0.0001)(25)$.

Active MMP-9 (aMMP-9). This form of MMP-9 is the functional form of the enzyme, which binds to different substrates of MMP-9 for degradation. The molecular weight of aMMP-9 is $87 \mathrm{kDa}$ (33). The plasma concentrations of aMMP-9 were found to be higher in breast cancer patients compared to healthy low risk participants $(p=0.015)(26)$. However, serum aMMP-9 concentrations did not differ between women with breast cancer and healthy women (27). Therefore, the association between aMMP-9 and breast cancer has not been clarified yet; further studies are needed to clarify the role of aMMP-9 in breast cancer.

Total MMP-9. Total MMP-9 (tMMP-9) consists of the proMMP-9 and active MMP-9 forms. Plasma MMP-9 levels were found to be lower in breast cancer patients than in healthy low risk women $(p=0.013)$ (26). In a small population, Katunina et al. (28) found that circulating levels of MMP-9 in serum were also lower in breast cancer cases than in controls $(p<0.05)$. However, one study found no differences in serum MMP-9 levels between breast cancer patients and healthy women $(p=0.177)(27)$. Taken together, it is possible that high tMMP-9 levels could be associated with lower breast cancer risk. 
Table I. Circulating levels of MMP-2 and MMP-9 and breast cancer risk.

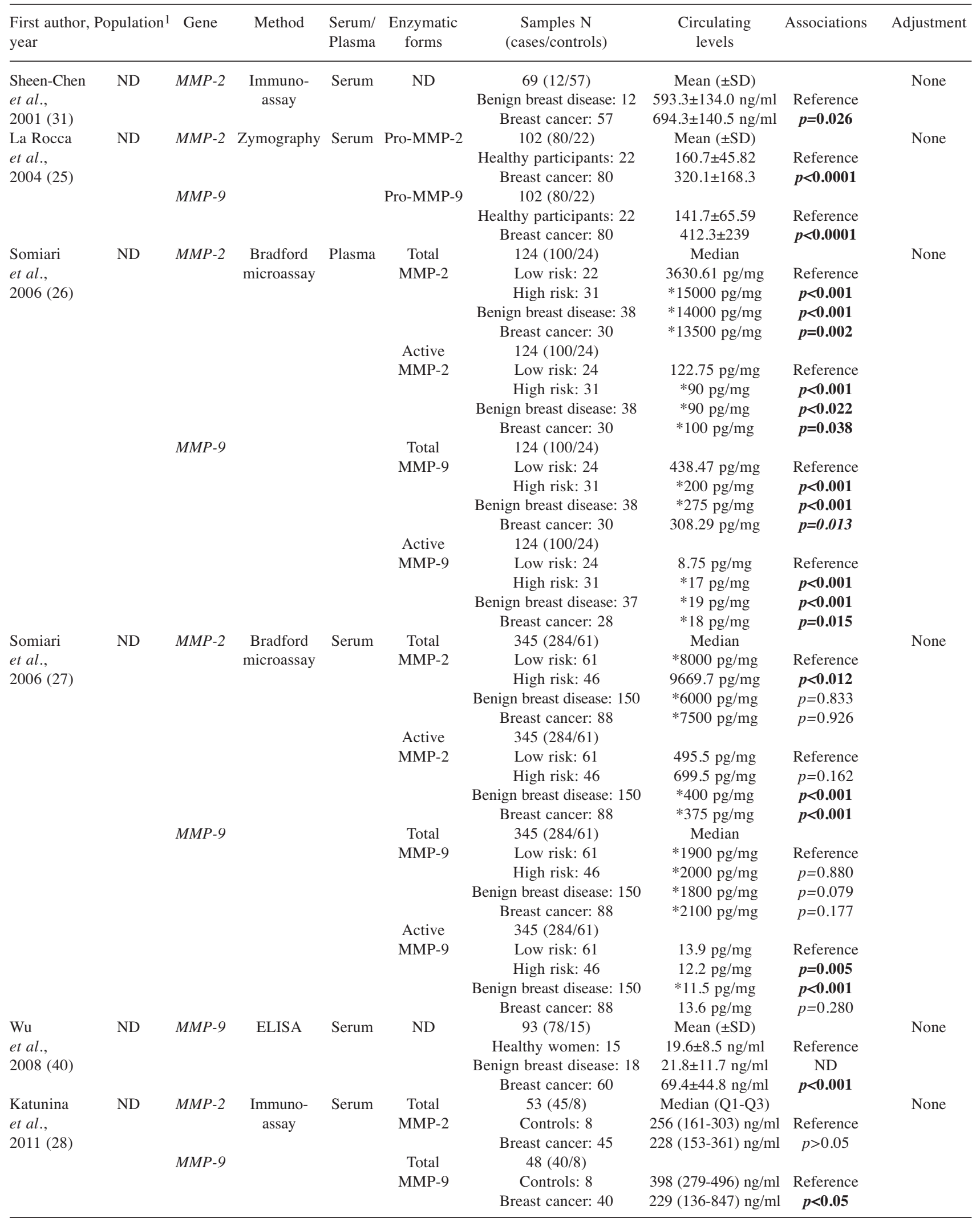


Table I. Continued

\begin{tabular}{|c|c|c|c|c|c|c|c|c|c|}
\hline $\begin{array}{l}\text { First author, } \\
\text { year }\end{array}$ & , Population ${ }^{1}$ & Gene & Method & $\begin{array}{l}\text { Serum/ } \\
\text { Plasma }\end{array}$ & $\begin{array}{l}\text { Enzymatic } \\
\text { forms }\end{array}$ & $\begin{array}{c}\text { Samples N } \\
\text { (cases/controls) }\end{array}$ & $\begin{array}{l}\text { Circulating } \\
\text { levels }\end{array}$ & Associations & Adjustment \\
\hline $\begin{array}{l}\text { Patel } \\
\text { et al., } \\
2011 \text { (32) }\end{array}$ & ND & MMP-9 & ELISA & Serum & ND & $\begin{array}{c}160(100 / 60) \\
\text { Healthy women: } 60 \\
\text { Benign breast disease: } 40 \\
\text { Breast cancer: } 60 \\
160(100 / 60) \\
\text { Healthy women: } 60 \\
\text { Benign breast disease: } 40 \\
\text { Breast cancer: } 60\end{array}$ & $\begin{array}{c}\text { Mean }( \pm \mathrm{SD}) \\
771.2 \pm 59.9 \mathrm{ng} / \mathrm{ml} \\
774.3 \pm 81.9 \mathrm{ng} / \mathrm{ml} \\
806.5 \pm 101.6 \mathrm{ng} / \mathrm{ml} \\
\\
272.5 \pm 41.6 \mathrm{ng} / \mathrm{ml} \\
288.3 \pm 37.3 \mathrm{ng} / \mathrm{ml} \\
371.9 \pm 47.1 \mathrm{ng} / \mathrm{ml}\end{array}$ & $\begin{array}{cc}1 & \text { Reference } \\
1 & p>0.05 \\
1 & \boldsymbol{p}<\mathbf{0 . 0 5} \\
& \\
1 & \text { Reference } \\
1 & p>0.05 \\
1 & \boldsymbol{p}<\mathbf{0 . 0 0 1}\end{array}$ & None \\
\hline $\begin{array}{l}\text { Kim } \\
\text { et al., } \\
2012(29)\end{array}$ & $\begin{array}{l}\text { Multiethnic } \\
\text { (Caucasian, } \\
\text { African- } \\
\text { American, } \\
\text { native } \\
\text { Hawaiians, } \\
\text { Japanese- } \\
\text { Americans) }\end{array}$ & $M M P-2$ & $\begin{array}{l}\text { Immuno- } \\
\text { fluorescence } \\
\text { assay }\end{array}$ & Plasma & $\begin{array}{l}\text { Total } \\
\text { MMP-2 }\end{array}$ & $\begin{array}{c}1462(731 / 731) \\
\text { Controls: } 731 \\
\text { Invasive breast } \\
\text { cancer: } 731\end{array}$ & $\begin{array}{c}\text { Mean }( \pm \mathrm{SD}) \\
20.5 \pm 0.21 \mathrm{pg} / \mathrm{ml} \\
20.4 \pm 1.79 \mathrm{pg} / \mathrm{ml}\end{array}$ & $\begin{array}{c}\text { Reference } \\
p=0.58\end{array}$ & None \\
\hline $\begin{array}{l}\text { Aroner } \\
\text { et al., } \\
2015(30)\end{array}$ & ND & $M M P-2$ & $\begin{array}{l}\text { Immuno- } \\
\text { assay }\end{array}$ & Plasma & $\begin{array}{c}\text { Total } \\
\text { MMP-2 }\end{array}$ & $\begin{array}{c}2272(1136 / 1136) \\
\text { Q1: } 284 / 309 \\
\text { Q2: } 284 / 264 \\
\text { Q3: 284/265 } \\
\text { Q4: 284/298 }\end{array}$ & $\begin{array}{c}\text { Quantile } \\
\text { Q1 } \leq 187.9 \\
\mathrm{ng} / \mathrm{ml} \\
\text { Q2: } 188.0-214.8 \\
\mathrm{ng} / \mathrm{ml} \\
\text { Q3: } 214.9-246.1 \\
\mathrm{ng} / \mathrm{ml} \\
\text { Q4: }>246.1 \\
\mathrm{ng} / \mathrm{ml}\end{array}$ & $\begin{array}{c}\mathrm{OR}=1.0 \\
\text { (reference) } \\
\mathrm{OR}=0.8 \\
(95 \% \mathrm{CI}=0.7-1.1) \\
\mathrm{OR}=0.9 \\
(95 \% \mathrm{CI}=0.7-1.1) \\
\mathrm{OR}=1.0 \\
(95 \% \mathrm{CI}=0.7-1.2) \\
p=0.890\end{array}$ & $\begin{array}{c}\text { Age, BMI, } \\
\text { age at } \\
\text { menarche, } \\
\text { menopausal, } \\
\text { current } \\
\text { alcohol } \\
\text { consumption, } \\
\text { PMH use, } \\
\text { parity, } \\
\text { family } \\
\text { history of } \\
\text { breast cancer, } \\
\text { history of } \\
\text { benign } \\
\text { breast } \\
\text { disease }\end{array}$ \\
\hline
\end{tabular}

MMP: Matrix metalloproteinase; ELISA: enzyme-linked immunosorbent assay; Q: quartile; M: mean; SD: standard deviation; BMI: body mass index; PMH: postmenopausal hormones; ND: not defined; CI: confidence interval. ${ }^{1}$ Study design: case-control; *Median estimated from box plots. Statistically significant differences are indicated in bold $(p<0.05)$.

No specific form. Two other studies evaluated the circulating levels of MMP-9, though without specifying the form, in breast cancer $(32,40)$. These studies demonstrated that circulating serum levels of MMP-9 were higher in breast cancer patients than healthy women $(p<0.05)$. These results are concordant with those observed for pro-MMP-9 levels and breast cancer risk.

\section{Polymorphisms Located in the MMP-9 Gene and Breast Cancer Risk}

Ten polymorphisms located in the MMP-9 gene have been studied in breast cancer risk, four of which have been the most investigated, namely: rs3918242, rs17576, rs2274756, rs2250889. Studies of the MMP-9 polymorphisms are described in Table II. rs3918242. The rs3918242 in the MMP-9 promoter is the most studied polymorphism for its relation to breast cancer risk. This polymorphism involves a $\mathrm{C}$ to $\mathrm{T}$ transition. The presence of the $\mathrm{T}$ allele leads to the loss of a nuclear repressor protein binding site and increases the expression of gelatinase B (41). The relationship between rs3918242 and breast cancer risk was unclear in the literature. The majority of studies did not find an association between this polymorphism and breast cancer risk in Caucasian (15) and Brazilian (16, 42) populations $(p>0.05)$. However, Chiranjeevi et al. (43) suggested a decreased risk of breast cancer in the Indian population using additive and recessive models, although deviation from Hardy-Weinberg equilibrium was observed for rs3918242 in this study. Similarly, Padala et al. (44) found that the TT genotype increased the risk of breast cancer, but also observed 
Table II. Polymorphisms in MMP-2 and -9 genes and breast cancer risk.

\begin{tabular}{|c|c|c|c|c|c|c|c|c|c|c|c|}
\hline Study & Population 1 & ${ }^{1}$ Cases & Controls & $\begin{array}{c}\text { Gene L } \\
\text { name } \\
\text { SNP }\end{array}$ & Localisation & HWE & $\begin{array}{c}\text { Genotype } \\
\text { distribution } \\
\text { Cases/Controls }\end{array}$ & $\begin{array}{l}\text { Genotyping } \\
\text { method }\end{array}$ & $\begin{array}{l}\text { Genotype/ } \\
\text { Allele }\end{array}$ & Analysis & $\begin{array}{c}\text { Adjustment/ } \\
\text { Matching }\end{array}$ \\
\hline $\begin{array}{l}\text { Zhou } \\
\text { et al., } \\
2004 \\
(14)\end{array}$ & Chinese & 462 & 509 & $\begin{array}{c}M M P-2 \\
\text { rs } 243865\end{array}$ & Promoter & YES & $\begin{array}{l}\text { CC } 381 / 349 \\
\text { CT } 79 / 154 \\
\text { TT } 2 / 6\end{array}$ & $\begin{array}{l}\text { PCR-based } \\
\text { DHPLC }\end{array}$ & $\begin{array}{c}\mathrm{CC} \\
\mathrm{CT}+\mathrm{TT}\end{array}$ & $\begin{array}{c}\mathrm{OR}=1.00 \\
\text { (reference) } \\
\mathrm{OR}=0.46 \\
(95 \% \mathrm{CI}=0.34-0.63) \\
\boldsymbol{p}<\mathbf{0 . 0 0 0 0 1}\end{array}$ & Age \\
\hline \multirow[t]{2}{*}{$\begin{array}{l}\text { Lei } \\
\text { et al., } \\
2007 \\
(15)\end{array}$} & \multirow[t]{2}{*}{ Swedish } & 949 & 948 & $\begin{array}{c}M M P-2 \\
\text { rs } 243865\end{array}$ & Promoter & YES & $\begin{array}{l}\text { CC } 520 / 520 \\
\text { CT } 359 / 359 \\
\text { TT 70/69 }\end{array}$ & $\begin{array}{c}\text { Taqman } \\
\text { assays }\end{array}$ & $\begin{array}{l}\mathrm{CC} \\
\mathrm{CT}\end{array}$ & $\begin{array}{c}\mathrm{OR}=1.00(\text { reference }) \\
\mathrm{OR}=1.00 \\
(95 \% \mathrm{CI}=0.83-1.21) \\
\mathrm{OR}=1.01 \\
(95 \% \mathrm{CI}=0.71-1.45)\end{array}$ & \multirow[t]{2}{*}{ None } \\
\hline & & 946 & 946 & $\begin{array}{c}M M P-9 \\
\text { rs3918242 }\end{array}$ & 2 Promoter & YES & $\begin{array}{l}\text { CC } 682 / 692 \\
\text { CT } 239 / 240 \\
\text { TT } 25 / 14\end{array}$ & Sequencing & $\begin{array}{l}\mathrm{CC} \\
\mathrm{CT} \\
\mathrm{TT}\end{array}$ & $\begin{array}{c}\mathrm{OR}=1.00 \text { (reference) } \\
\text { OR=1.01 } \\
(95 \% \mathrm{CI}: 0.82-1.24) \\
\text { OR }=1.88 \\
(95 \% \mathrm{CI}: 0.97-3.63)\end{array}$ & \\
\hline \multirow[t]{2}{*}{$\begin{array}{l}\text { Roehe } \\
\text { et al., } \\
2007 \\
(16)\end{array}$} & \multirow[t]{2}{*}{ Brazilian } & 89 & 100 & $\begin{array}{c}M M P-2 \\
\text { rs243865 }\end{array}$ & Promoter & YES & $\begin{array}{c}\text { CC } 63 / 66 \\
\text { CT } 21 / 32 \\
\text { TT } 5 / 2\end{array}$ & $\begin{array}{c}\text { DNA } \\
\text { Sequencing }\end{array}$ & $\begin{array}{l}\text { CC } \\
\text { CT } \\
\text { TT }\end{array}$ & $\begin{array}{c}\text { ND } \\
\text { ND } \\
\text { ND } \\
p=0.22\end{array}$ & \multirow[t]{2}{*}{ None } \\
\hline & & 96 & 100 & $\begin{array}{c}M M P-9 \\
\text { rs3918242 }\end{array}$ & 2 Promoter & YES & $\begin{array}{l}\text { CC } 76 / 83 \\
\text { CT } 20 / 15 \\
\text { TT } 0 / 2\end{array}$ & PCR-RFLP & $\begin{array}{l}\text { CC } \\
\text { CT } \\
\text { TT }\end{array}$ & $\begin{array}{c}\mathrm{ND} \\
\mathrm{ND} \\
\mathrm{ND} \\
p=0.23\end{array}$ & \\
\hline \multirow{9}{*}{$\begin{array}{l}\text { Delgado- } \\
\text { Enciso } \\
\text { et al., } \\
2008(17) \\
\text { Beeghly- } \\
\text { Fadiel } \\
\text { et al., } \\
2009 \text { (18) }\end{array}$} & Mexican & 90 & 96 & $\begin{array}{c}M M P-2 \\
\text { rs } 243865\end{array}$ & Promoter & YES & $\begin{array}{c}\text { CC } 63 / 50 \\
\text { CT } 25 / 42 \\
\text { TT } 2 / 4\end{array}$ & PCR & $\begin{array}{c}\mathrm{CT}+\mathrm{TT} \\
\mathrm{CC}\end{array}$ & $\begin{array}{c}\text { OR=1.00 (reference) } \\
\text { OR=2.15 } \\
(95 \% \mathrm{CI}=1.13-4.11) \\
\boldsymbol{p}=\mathbf{0 . 0 1}\end{array}$ & None \\
\hline & \multirow[t]{8}{*}{ Chinese } & \multirow[t]{8}{*}{3039} & \multirow[t]{8}{*}{3027} & $\begin{array}{c}M M P 2 \\
\text { rs } 1005912\end{array}$ & Promoter & YES & ND & Affymetrix & $\begin{array}{l}\text { TT } \\
\text { TA } \\
\text { AA }\end{array}$ & $\begin{array}{c}\mathrm{OR}=1.0(\text { reference }) \\
\mathrm{OR}=1.2(95 \% \mathrm{CI}=1.0-1.3) \\
\mathrm{OR}=1.1(95 \% \mathrm{CI}=0.9-1.3) \\
p=0.207\end{array}$ & $\begin{array}{c}\text { Age, } \\
\text { education }\end{array}$ \\
\hline & & & & rs1116195 & 5 Promoter & YES & ND & Sequenom & $\begin{array}{l}\text { AA } \\
\text { AT } \\
\text { TT }\end{array}$ & $\begin{array}{c}\mathrm{OR}=1.0(\text { reference }) \\
\mathrm{OR}=1.0(95 \% \mathrm{CI}=0.9-1.2) \\
\mathrm{OR}=1.2(95 \% \mathrm{CI}=1.0-1.4) \\
p=0.075\end{array}$ & \\
\hline & & & & rs11644561 & 1 Promoter & YES & ND & Affymetrix & $\begin{array}{l}\text { GG } \\
\text { GA } \\
\text { AA }\end{array}$ & $\begin{array}{c}\mathrm{OR}=1.0(\text { reference }) \\
\mathrm{OR}=0.9(95 \% \mathrm{CI}=0.8-1.1) \\
\mathrm{OR}=0.6(95 \% \mathrm{CI}=0.3-1.0) \\
p=0.098\end{array}$ & \\
\hline & & & & rs243867 & Promoter & YES & ND & Affymetrix & $\begin{array}{l}\text { AA } \\
\text { AG } \\
\text { GG }\end{array}$ & $\begin{array}{c}\mathrm{OR}=1.0(\text { reference }) \\
\mathrm{OR}=1.1(95 \% \mathrm{CI}=0.9-1.2) \\
\mathrm{OR}=1.1(95 \% \mathrm{CI}=0.9-1.3) \\
p=0.403\end{array}$ & \\
\hline & & & & rs11643630 & 0 Promoter & YES & ND & Affymetrix & $\begin{array}{l}\text { TT } \\
\text { TG } \\
\text { GG }\end{array}$ & $\begin{array}{c}\mathrm{OR}=1.00 \text { (reference) } \\
\mathrm{OR}=1.0(95 \% \mathrm{CI}=0.8-1.1) \\
\mathrm{OR}=0.8(95 \% \mathrm{CI}=0.7-1.0) \\
p=0.046\end{array}$ & \\
\hline & & & & rs243866 & Promoter & YES & ND & Affymetrix & $\begin{array}{l}\text { GG } \\
\text { GA } \\
\text { AA }\end{array}$ & $\begin{array}{c}\mathrm{OR}=1.00(\text { reference }) \\
\mathrm{OR}=1.0(95 \% \mathrm{CI}=0.9-1.2) \\
\mathrm{OR}=1.2(95 \% \mathrm{CI}=0.7-2.1) \\
p=0.602\end{array}$ & \\
\hline & & & & rs243865 & Promoter & YES & ND & Sequenom & $\begin{array}{l}\mathrm{CC} \\
\mathrm{CT} \\
\mathrm{TT}\end{array}$ & $\begin{array}{c}\mathrm{OR}=1.0(\text { reference }) \\
\mathrm{OR}=0.9(95 \% \mathrm{CI}=0.8-1.1) \\
\mathrm{OR}=1.4(95 \% \mathrm{CI}=0.9-2.4) \\
p=0.776\end{array}$ & \\
\hline & & & & rs243864 & Promoter & YES & ND & Affymetrix & $\begin{array}{l}\text { TT } \\
\text { TG } \\
\text { GG }\end{array}$ & $\begin{array}{c}\mathrm{OR}=1.0(\text { reference }) \\
\mathrm{OR}=1.0(95 \% \mathrm{CI}=0.9-1.2) \\
\mathrm{OR}=1.1(95 \% \mathrm{CI}=0.6-2.0) \\
p=0.782\end{array}$ & \\
\hline
\end{tabular}


Table II. Continued

\begin{tabular}{|c|c|c|c|c|c|c|c|c|c|c|}
\hline Study & Population ${ }^{1}$ Cases & Controls & $\begin{array}{l}\text { Gene L } \\
\text { name } \\
\text { SNP }\end{array}$ & Localisation & HWE & $\begin{array}{c}\text { Genotype } \\
\text { distribution } \\
\text { Cases/Controls }\end{array}$ & $\begin{array}{l}\text { Genotyping } \\
\text { method }\end{array}$ & $\begin{array}{c}\text { Genotype/ } \\
\text { Allele }\end{array}$ & Analysis & $\begin{array}{c}\text { Adjustment/ } \\
\text { Matching }\end{array}$ \\
\hline & & & rs2285053 & 3 Promoter & YES & ND & Sequenom & $\begin{array}{l}\mathrm{CC} \\
\mathrm{CT} \\
\mathrm{TT}\end{array}$ & $\begin{array}{c}\mathrm{OR}=1.0(\text { reference }) \\
\mathrm{OR}=1.2(95 \% \mathrm{CI}=1.0-1.4) \\
\mathrm{OR}=0.9(95 \% \mathrm{CI}=0.6-1.2) \\
p=0.436\end{array}$ & \\
\hline & & & rs 1477017 & 7 Intron 2 & YES & ND & $\begin{array}{l}\text { Affymetrix/ } \\
\text { Sequenom }\end{array}$ & $\begin{array}{l}\text { AA } \\
\text { AG } \\
\text { GG }\end{array}$ & $\begin{array}{c}\mathrm{OR}=1.0(\text { reference }) \\
\mathrm{OR}=1.0(95 \% \mathrm{CI}=0.9-1.2) \\
\mathrm{OR}=1.0(95 \% \mathrm{CI}=0.8-1.2) \\
p=0.833\end{array}$ & \\
\hline & & & rs865094 & Intron 2 & YES & ND & $\begin{array}{l}\text { Affymetrix/ } \\
\text { Sequenom }\end{array}$ & $\begin{array}{l}\text { AA } \\
\text { AG } \\
\text { GG }\end{array}$ & $\begin{array}{c}\mathrm{OR}=1.0(\text { reference }) \\
\mathrm{OR}=0.9(95 \% \mathrm{CI}=0.8-1.0) \\
\mathrm{OR}=1.1(95 \% \mathrm{CI}=0.9-1.4) \\
p=0.838\end{array}$ & \\
\hline & & & rs11646643 & 3 Intron 3 & YES & ND & Affymetrix & $\begin{array}{l}\text { AA } \\
\text { AG } \\
\text { GG }\end{array}$ & $\begin{array}{c}\mathrm{OR}=1.0(\text { reference }) \\
\mathrm{OR}=1.0(95 \% \mathrm{CI}=0.8-1.1) \\
\mathrm{OR}=1.1(95 \% \mathrm{CI}=0.7-1.6) \\
p=0.726\end{array}$ & \\
\hline & & & rs 1053605 & 5 Exon 5 & YES & ND & $\begin{array}{l}\text { Affymetrix/ } \\
\text { Sequenom }\end{array}$ & $\begin{array}{l}\text { CC } \\
\text { CT } \\
\text { TT }\end{array}$ & $\begin{array}{c}\mathrm{OR}=1.0(\text { reference }) \\
\mathrm{OR}=1.1(95 \% \mathrm{CI}=0.9-1.2) \\
\mathrm{OR}=0.8(95 \% \mathrm{CI}=0.4-1.3) \\
p=0.862\end{array}$ & \\
\hline & & & rs9302671 & Intron 5 & YES & ND & Affymetrix & $\begin{array}{l}\text { GG } \\
\text { GT } \\
\text { TT }\end{array}$ & $\begin{array}{c}\mathrm{OR}=1.0(\text { reference }) \\
\mathrm{OR}=1.0(95 \% \mathrm{CI}=0.8-1.1) \\
\mathrm{OR}=1.1(95 \% \mathrm{CI}=0.8-1.6) \\
p=0.936\end{array}$ & \\
\hline & & & rs2241145 & Intron 5 & YES & ND & Sequenom & $\begin{array}{l}\text { GG } \\
\text { GC } \\
\text { CC }\end{array}$ & $\begin{array}{c}\mathrm{OR}=1.0(\text { reference }) \\
\mathrm{OR}=1.0(95 \% \mathrm{CI}=0.8-1.2) \\
\mathrm{OR}=0.9(95 \% \mathrm{CI}=0.8-1.2) \\
p=0.613\end{array}$ & \\
\hline & & & rs2241146 & 5 Intron 5 & YES & ND & Sequenom & $\begin{array}{l}\text { GG } \\
\text { GA } \\
\text { AA }\end{array}$ & $\begin{array}{c}\mathrm{OR}=1.0(\text { reference }) \\
\mathrm{OR}=1.1(95 \% \mathrm{CI}=0.9-1.3) \\
\mathrm{OR}=1.0(95 \% \mathrm{CI}=0.7-1.5) \\
p=0.632\end{array}$ & \\
\hline & & & rs243849 & Exon 7 & YES & ND & Affymetrix & $\begin{array}{l}\text { CC } \\
\text { CT } \\
\text { TT }\end{array}$ & $\begin{array}{c}\mathrm{OR}=1.0(\text { reference }) \\
\mathrm{OR}=0.9(95 \% \mathrm{CI}=0.8-1.1) \\
\mathrm{OR}=1.1(95 \% \mathrm{CI}=0.8-1.6) \\
p=0.816\end{array}$ & \\
\hline & & & rs12599775 & 5 Intron 7 & YES & ND & Sequenom & $\begin{array}{l}\text { GG } \\
\text { GC } \\
\text { CC }\end{array}$ & $\begin{array}{c}\mathrm{OR}=1.0(\text { reference }) \\
\mathrm{OR}=1.1(95 \% \mathrm{CI}=0.9-1.4) \\
\mathrm{OR}=0.9(95 \% \mathrm{CI}=0.4-1.9) \\
p=0.453\end{array}$ & \\
\hline & & & rs 243847 & Intron 7 & YES & ND & $\begin{array}{l}\text { Affymetrix/ } \\
\text { Sequenom }\end{array}$ & $\begin{array}{l}\text { TT } \\
\text { TC } \\
\text { CC }\end{array}$ & $\begin{array}{c}\mathrm{OR}=1.0(\text { reference }) \\
\mathrm{OR}=1.1(95 \% \mathrm{CI}=0.9-1.2) \\
\mathrm{OR}=1.0(95 \% \mathrm{CI}=0.8-1.2) \\
p=0.881\end{array}$ & \\
\hline & & & rs2192852 & 2 Intron 7 & YES & ND & Sequenom & $\begin{array}{l}\text { AA } \\
\text { AG } \\
\text { GG }\end{array}$ & $\begin{array}{c}\mathrm{OR}=1.0(\text { reference }) \\
\mathrm{OR}=1.0(95 \% \mathrm{CI}=0.8-1.2) \\
\mathrm{OR}=0.9(95 \% \mathrm{CI}=0.7-1.2) \\
p=0.546\end{array}$ & \\
\hline & & & rs12923011 & 1 Intron 7 & YES & ND & Sequenom & $\begin{array}{l}\text { CC } \\
\text { CT } \\
\text { TT }\end{array}$ & $\begin{array}{c}\mathrm{OR}=1.0(\text { reference }) \\
\mathrm{OR}=0.9(95 \% \mathrm{CI}=0.7-1.1) \\
\mathrm{OR}=0.7(95 \% \mathrm{CI}=0.4-1.3) \\
p=0.118\end{array}$ & \\
\hline & & & rs 243845 & Intron 8 & YES & ND & Affymetrix & $\begin{array}{l}\text { GG } \\
\text { GA } \\
\text { AA }\end{array}$ & $\begin{array}{c}\mathrm{OR}=1.0(\text { reference }) \\
\mathrm{OR}=1.0(95 \% \mathrm{CI}=0.9-1.2) \\
\mathrm{OR}=1.0(95 \% \mathrm{CI}=0.8-1.2) \\
p=0.945\end{array}$ & \\
\hline & & & rs243844 & Intron 8 & YES & ND & Sequenom & $\begin{array}{l}\text { GG } \\
\text { GA } \\
\text { AA }\end{array}$ & $\begin{array}{c}\mathrm{OR}=1.0(\text { reference }) \\
\mathrm{OR}=1.0(95 \% \mathrm{CI}=0.8-1.2) \\
\mathrm{OR}=1.1(95 \% \mathrm{CI}=0.8-1.5) \\
p=0.604\end{array}$ & \\
\hline
\end{tabular}


Table II. Continued

\begin{tabular}{|c|c|c|c|c|c|c|c|c|c|c|c|}
\hline Study & Population 1 & Cases & Controls & $\begin{array}{l}\text { Sene L } \\
\text { name } \\
\text { SNP }\end{array}$ & Localisation & HWE & $\begin{array}{c}\text { Genotype } \\
\text { distribution } \\
\text { Cases/Controls }\end{array}$ & $\begin{array}{l}\text { Genotyping } \\
\text { method }\end{array}$ & $\begin{array}{c}\text { Genotype/ } \\
\text { Allele }\end{array}$ & Analysis & $\begin{array}{c}\text { Adjustment/ } \\
\text { Matching }\end{array}$ \\
\hline & & & & rs 2287074 & 4 Exon 9 & YES & ND & Sequenom & $\begin{array}{l}\text { GG } \\
\text { GA } \\
\text { AA }\end{array}$ & $\begin{array}{c}\mathrm{OR}=1.0(\text { reference }) \\
\mathrm{OR}=1.0(95 \% \mathrm{CI}=0.8-1.2) \\
\mathrm{OR}=0.8(95 \% \mathrm{CI}=0.5-1.1) \\
p=0.276\end{array}$ & \\
\hline & & & & rs243842 & Intron 9 & YES & ND & Affymetrix & $\begin{array}{l}\text { TT } \\
\text { TC } \\
\text { CC }\end{array}$ & $\begin{array}{c}\mathrm{OR}=1.0(\text { reference }) \\
\mathrm{OR}=1.0(95 \% \mathrm{CI}=0.9-1.2) \\
\mathrm{OR}=1.0(95 \% \mathrm{CI}=0.8-1.2) \\
p=0.882\end{array}$ & \\
\hline & & & & rs183112 & Intron 9 & YES & ND & Sequenom & $\begin{array}{l}\text { GG } \\
\text { GA } \\
\text { AA }\end{array}$ & $\begin{array}{c}\mathrm{OR}=1.0(\text { reference }) \\
\mathrm{OR}=1.0(95 \% \mathrm{CI}=0.8-1.2) \\
\mathrm{OR}=0.9(95 \% \mathrm{CI}=0.6-1.5) \\
p=0.874\end{array}$ & \\
\hline & & & & rs243839 & Intron 9 & YES & ND & $\begin{array}{c}\text { Affymetrix/ } \\
\text { Sequenom }\end{array}$ & $\begin{array}{l}\text { AA } \\
\text { AG } \\
\text { GG }\end{array}$ & $\begin{array}{c}\mathrm{OR}=1.0(\text { reference }) \\
\mathrm{OR}=1.0(95 \% \mathrm{CI}=0.9-1.1) \\
\mathrm{OR}=1.0(95 \% \mathrm{CI}=0.8-1.2) \\
p=0.924\end{array}$ & \\
\hline & & & & rs9923304 & 4 Intron 9 & YES & ND & Affymetrix & $\begin{array}{l}\text { CC } \\
\text { CT } \\
\text { TT }\end{array}$ & $\begin{array}{c}\mathrm{OR}=1.0(\text { reference }) \\
\mathrm{OR}=1.0(95 \% \mathrm{CI}=0.9-1.2) \\
\mathrm{OR}=0.9(95 \% \mathrm{CI}=0.7-1.2) \\
p=0.983\end{array}$ & \\
\hline & & & & rs 11639960 & 0 Intron 10 & YES & ND & $\begin{array}{c}\text { Affymetrix/ } \\
\text { Sequenom }\end{array}$ & $\begin{array}{l}\text { AA } \\
\text { AG } \\
\text { GG }\end{array}$ & $\begin{array}{c}\mathrm{OR}=1.0(\text { reference }) \\
\mathrm{OR}=1.0(95 \% \mathrm{CI}=0.9-1.1) \\
\mathrm{OR}=1.1(95 \% \mathrm{CI}=0.8-1.3) \\
p=0.889\end{array}$ & \\
\hline & & & & rs 243831 & 3' FR & YES & ND & Sequenom & $\begin{array}{l}\text { TT } \\
\text { TG } \\
\text { GG }\end{array}$ & $\begin{array}{c}\mathrm{OR}=1.0(\text { reference }) \\
\mathrm{OR}=0.8(95 \% \mathrm{CI}=0.7-1.0) \\
\mathrm{OR}=0.8(95 \% \mathrm{CI}=0.4-1.6) \\
p=0.113\end{array}$ & \\
\hline & & & & rs 12930259 & 93 ' FR & YES & ND & Sequenom & $\begin{array}{l}\text { TT } \\
\text { TC } \\
\text { CC }\end{array}$ & $\begin{array}{c}\mathrm{OR}=1.0(\text { reference }) \\
\mathrm{OR}=1.0(95 \% \mathrm{CI}=0.9-1.2) \\
\mathrm{OR}=1.0(95 \% \mathrm{CI}=0.7-1.3) \\
p=0.899\end{array}$ & \\
\hline & & & & rs2192853 & 3 3' FR & YES & ND & Sequenom & $\begin{array}{l}\text { AA } \\
\text { AG } \\
\text { GG }\end{array}$ & $\begin{array}{c}\mathrm{OR}=1.0(\text { reference }) \\
\mathrm{OR}=0.9(95 \% \mathrm{CI}=0.8-1.1) \\
\mathrm{OR}=1.0(95 \% \mathrm{CI}=0.7-1.3) \\
p=0.607\end{array}$ & \\
\hline & & & & rs1583587 & 7 3' FR & YES & ND & Affymetrix & $\begin{array}{l}\mathrm{GG} \\
\mathrm{GC} \\
\mathrm{CC}\end{array}$ & $\begin{array}{c}\mathrm{OR}=1.0(\text { reference }) \\
\mathrm{OR}=1.0(95 \% \mathrm{CI}=0.9-1.2) \\
\mathrm{OR}=1.1(95 \% \mathrm{CI}=0.8-1.20) \\
p=0.796\end{array}$ & \\
\hline & & & & rs8053806 & 6 3'FR & YES & ND & Affymetrix & $\begin{array}{l}\mathrm{CC} \\
\mathrm{CA} \\
\mathrm{AA}\end{array}$ & $\begin{array}{c}\mathrm{OR}=1.0(\text { reference }) \\
\mathrm{OR}=1.1(95 \% \mathrm{CI}=1.0-1.3) \\
\mathrm{OR}=1.1(95 \% \mathrm{CI}=0.9-1.5) \\
p=0.139\end{array}$ & \\
\hline & & & & rs 12708952 & 2 3'FR & YES & ND & Affymetrix & $\begin{array}{l}\text { GG } \\
\text { GC } \\
\text { CC }\end{array}$ & $\begin{array}{c}\mathrm{OR}=1.0(\text { reference }) \\
\mathrm{OR}=1.0(95 \% \mathrm{CI}=0.9-1.2) \\
\mathrm{OR}=1.0(95 \% \mathrm{CI}=0.8-1.2) \\
p=0.874\end{array}$ & \\
\hline & & & & rs 1583585 & 53 ' FR & YES & ND & Affymetrix & $\begin{array}{l}\text { GG } \\
\text { GA } \\
\text { AA }\end{array}$ & $\begin{array}{c}\mathrm{OR}=1.0(\text { reference }) \\
\mathrm{OR}=1.1(95 \% \mathrm{CI}=0.9-1.2) \\
\mathrm{OR}=1.1(95 \% \mathrm{CI}=0.9-1.5) \\
p=0.192\end{array}$ & \\
\hline \multirow[t]{2}{*}{$\begin{array}{l}\text { Beeghly- } \\
\text { Fadiel } \\
\text { et al., } \\
2011(46)\end{array}$} & Chinese & 2064 & 2081 & $\begin{array}{c}M M P 9 \\
\text { rs6065912 }\end{array}$ & 2 Promoter & YES & ND & Affymetrix & $\begin{array}{l}\text { AA } \\
\text { AG } \\
\text { GG }\end{array}$ & $\begin{array}{c}\mathrm{OR}=1.0(\text { reference }) \\
\mathrm{OR}=1.0(95 \% \mathrm{CI}=0.9-1.2) \\
\mathrm{OR}=0.8(95 \% \mathrm{CI}=0.3-2.0) \\
p=0.884\end{array}$ & \multirow[t]{2}{*}{$\begin{array}{c}\text { Age, } \\
\text { education }\end{array}$} \\
\hline & & 1056 & 1064 & rs4810482 & 2 Promoter & YES & ND & Sequenom & $\begin{array}{l}\text { CC } \\
\text { CT } \\
\text { TT }\end{array}$ & $\begin{array}{c}\mathrm{OR}=1.0(\text { reference }) \\
\mathrm{OR}=1.1(95 \% \mathrm{CI}=0.9-1.3) \\
\mathrm{OR}=1.0(95 \% \mathrm{CI}=0.7-1.3) \\
p=0.802\end{array}$ & \\
\hline
\end{tabular}


Table II. Continued

\begin{tabular}{|c|c|c|c|c|c|c|c|c|c|c|c|}
\hline Study & Population ${ }^{1}$ & ${ }^{1}$ Cases & Controls & $\begin{array}{l}\text { Sene L } \\
\text { name } \\
\text { SNP }\end{array}$ & Localisation & HWE & $\begin{array}{c}\text { Genotype } \\
\text { distribution } \\
\text { Cases/Controls }\end{array}$ & $\begin{array}{l}\text { Genotyping } \\
\text { method }\end{array}$ & $\begin{array}{c}\text { Genotype/ } \\
\text { Allele }\end{array}$ & Analysis & $\begin{array}{c}\text { Adjustment/ } \\
\text { Matching }\end{array}$ \\
\hline & & 1054 & 1064 & rs3918241 & 1 Promoter & YES & ND & Sequenom & $\begin{array}{l}\text { TT } \\
\text { TA } \\
\text { AA }\end{array}$ & $\begin{array}{c}\mathrm{OR}=1.0(\text { reference }) \\
\mathrm{OR}=1.1(95 \% \mathrm{CI}=0.9-1.3) \\
\mathrm{OR}=1.8(95 \% \mathrm{CI}=0.9-3.6) \\
p=0.116\end{array}$ & \\
\hline & & 1908 & 1919 & rs3918249 & 9 Promoter & YES & ND & Affymetrix & $\begin{array}{l}\text { TT } \\
\text { TC } \\
\text { CC }\end{array}$ & $\begin{array}{c}\mathrm{OR}=1.0(\text { reference }) \\
\mathrm{OR}=1.1(95 \% \mathrm{CI}=1.0-1.2) \\
\mathrm{OR}=1.1(95 \% \mathrm{CI}=0.9-1.5) \\
p=0.148\end{array}$ & \\
\hline & & 1058 & 1063 & rs 17576 & Exon 6 & YES & ND & Sequenom & $\begin{array}{l}\text { GG } \\
\text { GA } \\
\mathrm{AA}\end{array}$ & $\begin{array}{c}\mathrm{OR}=1.0 \text { (reference) } \\
\mathrm{OR}=1.0(95 \% \text { CI: } 0.9-1.2) \\
\mathrm{OR}=1.0(95 \% \text { CI: } 0.7-1.3) \\
p=0.905\end{array}$ & \\
\hline & & 1056 & 1062 & rs 2250889 & 9 Exon 10 & YES & ND & Sequenom & $\begin{array}{l}\mathrm{CC} \\
\mathrm{CG} \\
\mathrm{GG}\end{array}$ & $\begin{array}{c}\mathrm{OR}=1.0(\text { reference }) \\
\mathrm{OR}=1.0(95 \% \mathrm{CI}=0.9-1.2) \\
\mathrm{OR}=0.9(95 \% \mathrm{CI}=0.7-1.3) \\
p=0.990\end{array}$ & \\
\hline & & 1054 & 1064 & rs 2274756 & 6 Exon 12 & YES & ND & Sequenom & $\begin{array}{l}\text { GG } \\
\text { GA } \\
\text { AA }\end{array}$ & $\begin{array}{c}\mathrm{OR}=1.0(\text { reference }) \\
\mathrm{OR}=1.1(95 \% \mathrm{CI}=0.9-1.4) \\
\mathrm{OR}=2.0(95 \% \mathrm{CI}=1.0-4.0) \\
p=0.056\end{array}$ & \\
\hline $\begin{array}{l}\text { Saeed } \\
\text { et al., } \\
2013 \\
(19)\end{array}$ & $\begin{array}{c}\text { Saudi } \\
\text { population }\end{array}$ & 90 & 92 & $\begin{array}{c}M M P-2 \\
\text { rs } 243865\end{array}$ & Promoter & NO & $\begin{array}{c}\text { CC } 58 / 73 \\
\text { CT } 30 / 19 \\
\text { TT } 2 / 0\end{array}$ & PCR-RFLP & $\begin{array}{c}\mathrm{CC} \\
\mathrm{CT}+\mathrm{TT}\end{array}$ & $\begin{array}{c}\mathrm{OR}=1.00 \text { (reference) } \\
\mathrm{OR}=2.12 \\
(95 \% \mathrm{CI}=1.09-4.11) \\
\boldsymbol{p}=\mathbf{0 . 0 2 5}\end{array}$ & Age \\
\hline $\begin{array}{l}\text { Resler } \\
\text { et al., } \\
2013 \\
(47)\end{array}$ & Caucasian & 845 & 807 & $\begin{array}{l}M M P-9 \\
\text { rs17576 }\end{array}$ & Exon 6 & YES & $\begin{array}{l}\text { AA } 338 / 366 \\
\text { AG } 393 / 357 \\
\text { GG } 106 / 78\end{array}$ & $\begin{array}{l}\text { Illumina } \\
\text { GoldenGate } \\
\text { multiplex }\end{array}$ & $\begin{array}{l}\mathrm{AA} \\
\mathrm{AG} \\
\mathrm{GG}\end{array}$ & $\begin{array}{c}\mathrm{OR}=1.00(\text { reference }) \\
\mathrm{OR}=1.21(95 \% \mathrm{CI}=1.04-1.40) \\
\mathrm{OR}=1.46(95 \% \mathrm{CI}=1.08-1.96) \\
\boldsymbol{p}=\mathbf{0 . 0 1}\end{array}$ & $\begin{array}{l}\text { Age- } \\
\text { matched }\end{array}$ \\
\hline & & & & rs 2274756 & 6 Exon 12 & YES & $\begin{array}{c}\text { GG } 338 / 366 \\
\text { GA } 393 / 357 \\
\text { AA } 106 / 78\end{array}$ & & $\begin{array}{ll}\text { GG } \\
\text { GA } \\
\text { AA }\end{array}$ & $\begin{array}{c}\mathrm{OR}=1.00(\text { reference }) \\
\mathrm{OR}=1.14(95 \% \mathrm{CI}=0.94-1.40) \\
\mathrm{OR}=1.30(95 \% \mathrm{CI}=0.88-1.96) \\
p=0.19\end{array}$ & \\
\hline & & & & rs3918262 & 2 Intron & YES & $\begin{array}{c}\text { AA } 327 / 518 \\
\text { AG } 195 / 246 \\
\text { GG } 35 / 31\end{array}$ & & $\begin{array}{l}\text { AA } \\
\text { AG O } \\
\text { GG }\end{array}$ & $\begin{array}{c}\mathrm{OR}=1.00(\text { reference }) \\
\mathrm{OR}=1.18(95 \% \mathrm{CI}=1.00-1.40) \\
\mathrm{OR}=1.39(95 \% \mathrm{CI}=1.00-1.96) \\
p=0.05\end{array}$ & \\
\hline $\begin{array}{l}\text { Zagouri } \\
\text { et al., } \\
2013 \\
(20)\end{array}$ & Greek & 113 & 124 & $\begin{array}{c}M M P-2 \\
\text { rs } 243865\end{array}$ & Promoter & YES & $\begin{array}{c}\text { CC } 63 / 83 \\
\text { CT } 41 / 37 \\
\text { TT } 9 / 4\end{array}$ & $\begin{array}{l}\text { Nucleopsin } \\
\text { Tissue } \\
\text { kit }\end{array}$ & $\begin{array}{l}\mathrm{CC} \\
\mathrm{CT} \\
\mathrm{TT}\end{array}$ & $\begin{array}{c}\mathrm{OR}=1.00(\text { reference }) \\
\mathrm{OR}=1.48(95 \% \mathrm{CI}=0.84-2.58) \\
\mathrm{OR}=2.90 \\
(95 \% \mathrm{CI}=0.82-10.29)\end{array}$ & $\begin{array}{c}\text { Age, } \\
\text { smoking, } \\
\text { alcohol, } \\
\text { BMI, } \\
\text { menopausal } \\
\text { status, } \\
\text { age at } \\
\text { menarche, } \\
\text { education }\end{array}$ \\
\hline \multirow[t]{3}{*}{$\begin{array}{l}\text { Slattery } \\
\text { et al., } \\
2013 \\
(21)\end{array}$} & \multirow[t]{3}{*}{$\begin{array}{c}\text { Caucasian- } \\
\text { Hispanic }\end{array}$} & \multirow[t]{3}{*}{3592} & 4183 & $\begin{array}{c}M M P-2 \\
\text { rs } 243865\end{array}$ & Promoter & YES & $\begin{array}{c}\text { CC+CT } \\
3183 / 3646 \\
\text { TT } 386 / 504\end{array}$ & $\begin{array}{l}\text { Multiplexed } \\
\text { bead } \\
\text { array assay }\end{array}$ & $\begin{array}{c}\mathrm{CC}+\mathrm{CT} \\
\mathrm{TT}\end{array}$ & $\begin{array}{c}\mathrm{OR}=1.00 \text { (reference) } \\
\mathrm{OR}=0.84 \\
(95 \% \mathrm{CI}=0.73-0.97) \\
*_{p}=0.08\end{array}$ & $\begin{array}{l}\text { Age, BMI, } \\
\text { parity, } \\
\text { genetic } \\
\text { admixture, }\end{array}$ \\
\hline & & & & rs11541998 & $8 \quad$ ND & YES & $\begin{array}{c}\text { CC } 2957 / 3547 \\
\text { CG+GG } \\
610 / 600\end{array}$ & & $\begin{array}{c}\mathrm{CC} \\
\mathrm{CG}+\mathrm{GG}\end{array}$ & $\begin{array}{c}\mathrm{OR}=1.00 \text { (reference) } \\
\mathrm{OR}=1.16 \\
(95 \% \mathrm{CI}=1.02-1.31) \\
* p=0.08\end{array}$ & $\begin{array}{l}\text { study } \\
\text { center } \\
\text { (Mexico } \\
\text { city, }\end{array}$ \\
\hline & & & & $\begin{array}{c}M M P-9 \\
\text { rs3787268 }\end{array}$ & $8^{\text {Intron }}$ & YES & $\begin{array}{c}\text { GG } 2479 / 2930 \\
\text { GA+AA } \\
1074 / 1202\end{array}$ & & $\begin{array}{c}\text { GG } \\
\text { GA+AA }\end{array}$ & $\begin{array}{c}\mathrm{OR}=1.00 \text { (reference) } \\
\mathrm{OR}=1.00 \\
(95 \% \mathrm{CI}=0.91-1.11) \\
*_{p}=0.96\end{array}$ & $\begin{array}{c}\text { San } \\
\text { Francisco } \\
\text { Bay Area, } \\
4 \text { corner's) }\end{array}$ \\
\hline
\end{tabular}

Table II. Continued 
Table II. Continued

\begin{tabular}{|c|c|c|c|c|c|c|c|c|c|c|c|}
\hline Study & Population ${ }^{1}$ & Cases & Controls & $\begin{array}{l}\text { sene L } \\
\text { name } \\
\text { SNP }\end{array}$ & Localisation & HWE & $\begin{array}{c}\text { Genotype } \\
\text { distribution } \\
\text { Cases/Controls }\end{array}$ & $\begin{array}{l}\text { Genotyping } \\
\text { method }\end{array}$ & $\begin{array}{c}\text { Genotype/ } \\
\text { Allele }\end{array}$ & Analysis & $\begin{array}{c}\text { Adjustment/ } \\
\text { Matching }\end{array}$ \\
\hline $\begin{array}{l}\text { Chiranjeevi } \\
\text { et al., } \\
2014 \\
(43)\end{array}$ & vi Indian & 200 & 191 & $\begin{array}{c}M M P-9 \\
\text { rs3918242 }\end{array}$ & 2 Promoter & NO & $\begin{array}{l}\text { CC } 73 / 86 \\
\text { CT } 66 / 68 \\
\text { TT } 61 / 37\end{array}$ & AS-PCR & $\begin{array}{c}\mathrm{CC} \\
\mathrm{CT} \\
\mathrm{TT} \\
\mathrm{CC} \\
\mathrm{CT}+\mathrm{TT} \\
\\
\mathrm{CT}+\mathrm{CC} \\
\mathrm{TT} \quad \mathrm{O}\end{array}$ & $\begin{array}{c}\mathrm{OR}=1.00 \text { (reference) } \\
\mathrm{OR}=0.87(95 \% \mathrm{CI}=0.55-0.38) \\
\mathrm{OR}=0.51(95 \% \mathrm{CI}=0.38-0.86) \\
\mathrm{OR}=1.00(\text { reference }) \\
\mathrm{OR}=0.70 \\
(95 \% \mathrm{CI}=0.46-1.05) \\
p=0.1068 \\
\mathrm{OR}=1.00(\text { reference }) \\
\mathrm{OR}=0.54(95 \% \mathrm{CI}=0.34-0.87) \\
\boldsymbol{p}=\mathbf{0 . 0 1 5}\end{array}$ & None \\
\hline $\begin{array}{l}\text { Yari K. } \\
\text { et al., } \\
2014(38)\end{array}$ & Iranian & 98 & 135 & $\begin{array}{c}M M P-2 \\
\text { rs } 2285053\end{array}$ & 3 Promoter & YES & $\begin{array}{l}\text { CC } 70 / 80 \\
\text { CT } 28 / 52 \\
\text { TT } 0 / 3\end{array}$ & PCR-RFLP & $\begin{array}{l}\mathrm{T} \\
\mathrm{C}\end{array}$ & $\begin{array}{c}\mathrm{OR}=1.00(\text { reference }) \\
\mathrm{OR}=1.64(95 \% \mathrm{CI}=1.01-2.7) \\
\boldsymbol{p = 0 . 0 4 9}\end{array}$ & None \\
\hline $\begin{array}{l}\text { Chahil } \\
\text { et al., } \\
2015(48)\end{array}$ & Malaysian & 80 & 81 & $\begin{array}{l}M M P-9 \\
\text { rs } 17576\end{array}$ & Exon 6 & NO & $\begin{array}{c}\text { AA } 4 / 15 \\
\text { AG } 26 / 29 \\
\text { GG } 50 / 37\end{array}$ & $\begin{array}{l}\text { Illumina } \\
\text { GoldenGate }\end{array}$ & $\begin{array}{l}\text { AA } \\
\text { AG } \\
\text { GG }\end{array}$ & $\begin{array}{c}\mathrm{OR}=1.00 \text { (reference) } \\
\mathrm{OR}=3.14 \\
(95 \% \mathrm{CI}=0.92-10.74) \\
\mathrm{OR}=4.73 \\
(95 \% \mathrm{CI}=1.44-15.54)\end{array}$ & Age \\
\hline & & 80 & $80 \quad 1$ & rs 2250889 & 9 Exon 10 & NO & $\begin{array}{c}\text { CC } 1 / 8 \\
\text { CG } 18 / 27 \\
\text { GG } 61 / 45\end{array}$ & & $\begin{array}{l}\mathrm{CC} \\
\mathrm{CG} \\
\mathrm{GG}\end{array}$ & $\begin{array}{c}\mathrm{OR}=1.00(\text { reference }) \\
\mathrm{OR}=5.33 \\
(95 \% \mathrm{CI}=0.61-46.37) \\
\mathrm{OR}=10.84 \\
(95 \% \mathrm{CI}=1.31-89.83)\end{array}$ & \\
\hline $\begin{array}{l}\text { Néjima F. } \\
\text { et al., } \\
2015(22)\end{array}$ & Tunisian & 210 & 250 & $\begin{array}{c}M M P-2 \\
\text { rs } 243865\end{array}$ & Promoter & YES & $\begin{array}{l}\text { CC } 118 / 97 \\
\text { CT } 69 / 105 \\
\text { TT } 23 / 48\end{array}$ & RT-PCR & $\begin{array}{l}\mathrm{CT}+\mathrm{TT} \\
\mathrm{CC}\end{array}$ & $\begin{array}{c}\mathrm{OR}=1.00(\text { reference }) \\
\mathrm{OR}=2.54 \\
(95 \% \mathrm{CI}=1.39-4.06)\end{array}$ & None \\
\hline $\begin{array}{l}\text { Rahimi Z. } \\
\text { et al., } \\
2015(45)\end{array}$ & $\begin{array}{l}\text { Iranian } \\
\text { (Kurdish) }\end{array}$ & 101 & 104 & $\begin{array}{c}M M P-9 \\
\text { rs3918242 }\end{array}$ & 2 Promoter & YES & $\begin{array}{l}\text { CC } 68 / 84 \\
\text { CT } 31 / 19 \\
\text { TT } 2 / 1\end{array}$ & PCR-RFLP & $\begin{array}{c}\mathrm{CC} \\
\mathrm{CT} \\
\mathrm{TT} \\
\mathrm{CC} \\
\mathrm{CT}+\mathrm{TT} \\
\mathrm{CT}+\mathrm{CC} \\
\mathrm{TT} \\
\mathrm{CC}+\mathrm{TT} \\
\mathrm{CT}\end{array}$ & $\begin{array}{c}\mathrm{OR}=1.00 \text { (reference) } \\
\mathrm{OR}=2.02(95 \% \mathrm{CI}=1.05-3.88) \\
\mathrm{OR}=1.57(95 \% \mathrm{CI}=1.57-5.28) \\
\mathrm{OR}=1.00(\text { reference }) \\
\mathrm{OR}=2.04 \\
(95 \% \mathrm{CI}=1.07-3.87) \\
\mathrm{OR}=1.00 \text { (reference) } \\
\mathrm{OR}=2.08 \\
(95 \% \mathrm{CI}=0.18-23.31) \\
\mathrm{OR}=1.00(\text { reference }) \\
\mathrm{OR}=1.98 \\
(95 \% \mathrm{CI}=1.03-3.81)\end{array}$ & None \\
\hline $\begin{array}{l}\text { Padala } \\
\text { et al., } \\
2017 \text { (44) }\end{array}$ & Indian & 300 & 300 & $\begin{array}{c}M M P-9 \\
\text { rs3918242 }\end{array}$ & Promoter & NO & $\begin{array}{l}\text { CC } 121 / 150 \\
\text { CT } 107 / 101 \\
\text { TT } 72 / 49\end{array}$ & AS-PCR & $\begin{array}{ll}\mathrm{CC} & \\
\mathrm{CT} & \mathrm{O} \\
\mathrm{TT} & \mathrm{O}\end{array}$ & $\begin{aligned} \mathrm{OR} & =1.00(\text { reference }) \\
\mathrm{OR} & =1.31(95 \% \mathrm{CI}=0.91-1.88) \\
\mathrm{OR} & =1.82(95 \% \mathrm{CI}=1.18-2.81)\end{aligned}$ & None \\
\hline $\begin{array}{l}\text { Felizi } \\
\text { et al., } \\
2018(42)\end{array}$ & Brazilian & 148 & 245 & $\begin{array}{c}M M P-9 \\
\text { rs3918242 }\end{array}$ & Promoter & YES & $\begin{array}{c}\text { CC } 115 / 186 \\
\text { CT+TT } 24 / 45\end{array}$ & PCR-RFLP & $\begin{array}{c}\mathrm{CC} \\
\mathrm{CT}+\mathrm{TT}\end{array}$ & $\begin{array}{c}\mathrm{OR}=1.00 \text { (reference) } \\
\text { OR=1.16 } \\
(95 \% \text { CI: } 0.66-2.00) \\
p=0.5964\end{array}$ & None \\
\hline \multirow[t]{4}{*}{$\begin{array}{l}\text { Habel } \\
\text { et al., } \\
2019 \text { (23) }\end{array}$} & Tunisian & 430 & 498 & $\begin{array}{c}M M P-2 \\
\text { rs243864 }\end{array}$ & Promoter & YES & $\begin{array}{l}\text { TT } 297 / 366 \\
\text { TG } 112 / 120 \\
\text { GG } 18 / 12\end{array}$ & $\begin{array}{l}\text { TaqMan } \\
\text { assays }\end{array}$ & $\begin{array}{l}\mathrm{G} \\
\mathrm{T}\end{array}$ & $\begin{array}{c}\mathrm{OR}=1.00 \text { (reference) } \\
\mathrm{OR}=1.24 \\
(95 \% \mathrm{CI}=0.97-1.59)\end{array}$ & None \\
\hline & & & & rs243865 & Promoter & YES & $\begin{array}{l}\text { CC } 291 / 350 \\
\text { CT } 122 / 108 \\
\text { TT } 14 / 40\end{array}$ & & $\begin{array}{l}\mathrm{C} \\
\mathrm{T}\end{array}$ & $\begin{array}{c}\mathrm{OR}=1.00 \text { (reference) } \\
\mathrm{OR}=0.92 \\
(95 \% \mathrm{C}=0.73-1.17)\end{array}$ & \\
\hline & & & & rs243866 & Promoter & YES & $\begin{array}{l}\text { GG } 251 / 352 \\
\text { GT } 101 / 120 \\
\text { TT } 11 / 8\end{array}$ & & $\begin{array}{l}\mathrm{G} \\
\mathrm{A}\end{array}$ & $\begin{array}{c}\mathrm{OR}=1.00(\text { reference }) \\
\mathrm{OR}=0.24 \\
(95 \% \mathrm{CI}=0.95-1.61)\end{array}$ & \\
\hline & & & & rs 2285053 & 3 Promoter & YES & $\begin{array}{l}\text { CC } 303 / 307 \\
\text { CT } 89 / 169 \\
\text { TT } 11 / 22\end{array}$ & & $\begin{array}{l}\mathrm{C} \\
\mathrm{T}\end{array}$ & $\begin{array}{c}\mathrm{OR}=1.00 \text { (reference) } \\
\mathrm{OR}=0.59 \\
(95 \% \mathrm{CI}=0.46-0.75)\end{array}$ & \\
\hline
\end{tabular}

HWE: Hardy-Weinberg equilibrium; MMP: matrix metalloproteinase; SNP: single nucleotide polymorphisms; DNA: deoxyribonucleic acid; PCRDHPLC: polymerase chain reaction-denaturing high performance liquid chromatography; PCR-RFLP: polymerase chain reaction-restriction fragment length polymorphism; RT-PCR: reverse transcription-polymerase chain reaction; AS-PCR: allele specific-polymerase chain reaction; ND: not defined; OR: odds ratio; CI: confidence interval; BMI: body mass index. Statistically significant differences are indicated in bold italics $(p<0.05)$. ${ }^{1}$ Study design: case-control; * $p$-values are adjusted for multiple comparisons. 
deviation from Hardy-Weinberg equilibrium for rs3918242. One study conducted in the Iranian population showed an increased risk of breast cancer using additive and dominant models (45). In total, literature evidence suggests that rs3918242 in MMP-9 does not influence breast cancer risk.

rs2274756. This polymorphism is a $\mathrm{G} \rightarrow \mathrm{A}$ transition in exon 12 of MMP-9 gene. In previous studies, it was not associated with breast cancer risk in Asian $(p=0.056)$ (46) and Caucasian $(p=0.19)(47)$ populations.

rs2250889. The rs2250889 polymorphism is a $\mathrm{G} \rightarrow \mathrm{C}$ transition located in exon 10. Two studies in the Asian population have demonstrated conflicting results. BeeghlyFadiel et al. (46) did not find any association between rs2250889 and breast cancer risk. On the other hand, Chachil et al. (48) analyzed a small population and showed that the GG genotype increased the risk of breast cancer $(\mathrm{OR}=10.84$; 95\% CI=1.31-89.83) compared to CC.

rs 17576. The rs17576 is an $\mathrm{A} \rightarrow \mathrm{G}$ transition located in exon 6. The $\mathrm{G}$ allele alters protein conformation and changes substrate-binding and enzyme activity (49). The GA and GG genotypes of rs17576 compared to AA were associated with increased breast cancer risk in Caucasian populations (GA: $\mathrm{OR}=1.21$; $95 \% \mathrm{CI}=1.04-1.40$; GG: $\mathrm{OR}=1.46$; $95 \% \mathrm{CI}=1.08$ $1.96 ; p=0.01)$ (47). The GG genotype was also associated with increased breast cancer risk compared to AA in Asian populations $(\mathrm{OR}=4.73 ; 95 \% \mathrm{CI}=1.44-15.54)$ (48), but deviation from Hardy-Weinberg equilibrium was observed in this study. However, conflicting evidence was presented in a study by Beeghly-Fadiel et al., who did not find any association between rs17576 and breast cancer risk $(p=0.905)$ in an Asian population (46).

Other polymorphisms in MMP-9. Some other polymorphisms in MMP-9 [rs6065912 (46), rs4810482 (46), rs3918241 (46), rs3918249 (46), rs3918262 (46), rs3787268 (21)] have been investigated in relation to breast cancer risk. No evidence has been found to support an association between those polymorphisms and breast cancer risk.

Haplotypes in MMP-9. To our knowledge, one study performed haplotype analysis for polymorphisms (rs6065912, rs4810482, rs3918241, rs3918249, rs17576, rs2250889, rs2274756) in MMP-9 gene, but no significant associations were revealed (46).

\section{Conclusion}

Review of evidence on the relation between 37 polymorphisms located in $M M P$-2 gene and breast cancer risk showed that most of them were not associated with breast cancer risk. Among these polymorphisms, the T allele of rs243865 and rs 2285053 was the only one that has been associated with reduced expression of $M M P-2$ as well as with decreased breast cancer risk. However, functional analyses of rs2285053 polymorphism are required. These observations are consistent with the results of circulating levels of latent or unspecified forms of MMP-2 that appear to be higher in breast cancer patients than in healthy women. Conversely, aMMP-2 levels appeared to be lower in breast cancer cases than in healthy women. Since it is unclear why levels of pro-MMP-2 and aMMP-2 are associated in opposite directions with breast cancer risk, it would be useful for future studies to clarify why the direction of this association depends on the forms of MMP-2.

Concerning the 10 studied polymorphisms located in $M M P$ 9, only rs17576 G allele was reported to influence breast cancer risk. However, only a few studies have evaluated this association. Additional, functional analyses and studies with homogenous populations are required. Similar to MMP-2, circulating levels of latent or unspecified forms of MMP-9 were higher in breast cancer patients than healthy women, while the opposite was observed for total MMP-9 levels. Thus, several analyses are necessary to clarify why different forms of MMP-9 are differentially associated with breast cancer risk. Furthermore, most studies so far have measured MMP-9 in serum. However, the literature suggests that serum samples are not appropriate to assess MMP-9 concentrations $(50,51)$. Hence, future studies should focus on plasma levels in order to investigate the association between different forms of circulating MMP-9 and breast cancer risk.

\section{Conflicts of Interest}

The Authors declare that they have no competing interests.

\section{Authors' Contributions}

SGD and CD designed the review, wrote the manuscript and analyzed results of literature research. SGD, CD and SLC reviewed the article.

\section{References}

1 Nagase H, Visse R and Murphy G: Structure and function of matrix metalloproteinases and timps. Cardiovasc Res 69(3): 562-573, 2006. PMID: 16405877. DOI: 10.1016/j.cardiores.2005.12.002

2 Murphy G and Nagase H: Progress in matrix metalloproteinase research. Mol Aspects Med 29(5): 290-308, 2008. PMID: 18619669. DOI: 10.1016/j.mam.2008.05.002

3 Cui N, Hu M and Khalil RA: Biochemical and biological attributes of matrix metalloproteinases. In: Prog mol biol transl sci. Elsevier, pp. 1-73, 2017.

4 Nelson AR, Fingleton B, Rothenberg ML and Matrisian LM: Matrix metalloproteinases: Biologic activity and clinical implications. J Clin Oncol 18(5): 1135-1149, 2000. PMID: 10694567. DOI: $10.1200 / J C O .2000 .18 .5 .1135$ 
5 Gillard JA, Reed MW, Buttle D, Cross SS and Brown NJ: Matrix metalloproteinase activity and immunohistochemical profile of matrix metalloproteinase- 2 and -9 and tissue inhibitor of metalloproteinase-1 during human dermal wound healing. Wound Repair Regen 12(3): 295-304, 2004. PMID: 15225208. DOI: $10.1111 /$ j.1067-1927.2004.012314.x

6 Werb Z, Ashkenas J, MacAuley A and Wiesen J: Extracellular matrix remodeling as a regulator of stromal-epithelial interactions during mammary gland development, involution and carcinogenesis. Braz J Med Biol Res 29(9): 1087-1097, 1996. PMID: 9181050.

7 Duffy MJ, Maguire TM, Hill A, McDermott E and O'Higgins $\mathrm{N}$ : Metalloproteinases: role in breast carcinogenesis, invasion and metastasis. Breast Cancer Res 2(4): 252-257, 2000. PMID: 11250717. DOI: $10.1186 /$ bcr65

8 Roy R, Morad G, Jedinak A and Moses MA: Metalloproteinases and their roles in human cancer. Anat Rec (Hoboken), 2019. PMID: 31168956. DOI: 10.1002/ar.24188

9 Coussens LM and Werb Z: Inflammation and cancer. Nature 420(6917): 860-867, 2002. PMID: 12490959. DOI: $10.1038 /$ nature 01322

10 Chambers AF and Matrisian LM: Changing views of the role of matrix metalloproteinases in metastasis. J Natl Cancer Inst 89(17): 1260-1270, 1997. PMID: 9293916. DOI: 10.1093/jnci/ 89.17 .1260

11 Cockett MI, Murphy G, Birch M, O’Connell J, Crabbe T, Millican A, Hart I and Docherty A: Matrix metalloproteinases and metastatic cancer. Biochem Soc Symp 63: 295-313, 1998. PMID: 9513731.

12 Aparicio T and Lehy T: Matrix metalloproteases in digestive pathology. Gastroenterol Clin Biol 23(3): 330-341, 1999. PMID: 10384335 .

13 Dofara SG, Chang SL and Diorio C: Association between the polymorphisms in MMP-2 and MMP-9 with adiposity and mammographic features. Breast Cancer Res Treat, 2020. PMID: 32394348. DOI: 10.1007/s10549-020-05651-0

14 Zhou Y, Yu C, Miao X, Tan W, Liang G, Xiong P, Sun T and Lin $\mathrm{D}$ : Substantial reduction in risk of breast cancer associated with genetic polymorphisms in the promoters of the matrix metalloproteinase-2 and tissue inhibitor of metalloproteinase-2 genes. Carcinogenesis 25(3): 399-404, 2004. PMID: 14604886. DOI: $10.1093 /$ carcin/bgh020

15 Lei H, Hemminki K, Altieri A, Johansson R, Enquist K, Hallmans G, Lenner P and Forsti A: Promoter polymorphisms in matrix metalloproteinases and their inhibitors: Few associations with breast cancer susceptibility and progression. Breast Cancer Res Treat 103(1): 61-69, 2007. PMID: 17033924. DOI: $10.1007 / \mathrm{s} 10549-006-9345-2$

16 Roehe AV, Frazzon APG, Agnes G, Damin AP, Hartman AA and Graudenz MS: Detection of polymorphisms in the promoters of matrix metalloproteinases 2 and 9 genes in breast cancer in south brazil: Preliminary results. Breast Cancer Res Treat 102(1): 123124, 2007. PMID: 17260100. DOI: 10.1007/s10549-006-9273-1

17 Delgado-Enciso I, Cepeda-Lopez FR, Monrroy-Guizar EA, Bautista-Lam JR, Andrade-Soto M, Jonguitud-Olguin G, Rodriguez-Hernandez A, Anaya-Ventura A, Baltazar-Rodriguez LM and Orozco-Ruiz M: Matrix metalloproteinase-2 promoter polymorphism is associated with breast cancer in a mexican population. Gynecol Obstet Invest 65(1): 68-72, 2008. PMID: 17851253. DOI: $10.1159 / 000108282$
18 Beeghly-Fadiel A, Lu W, Long J-R, Shu X-o, Zheng Y, Cai Q, Gao Y-T and Zheng W: Matrix metalloproteinase-2 polymorphisms and breast cancer susceptibility. Cancer Epidemiol Biomarkers Prev 18(6): 1770-1776, 2009. PMID: 19454611. DOI: 10.1158/1055-9965.EPI-09-0125

19 Saeed HM, Alanazi MS, Alshahrani O, Parine NR, Alabdulkarim HA and Shalaby MA: Matrix metalloproteinase-2 C(-1306)T promoter polymorphism and breast cancer risk in the Saudi population. Acta Biochim Pol 60(3): 405-409, 2013. PMID: 24051440 .

20 Zagouri F, Sergentanis TN, Gazouli M, Dimitrakakis C, Tsigginou A, Papaspyrou I, Chrysikos D, Lymperi M, Zografos GC, Antsaklis A, Dimopoulos M-A and Papadimitriou CA: MMP-2 $-1306 \mathrm{C}>\mathrm{T}$ polymorphism in breast cancer: A case-control study in a south european population. Mol Biol Rep 40(8): 5035-5040, 2013. PMID: 23661021. DOI: 10.1007/s11033-013-2604-5

21 Slattery ML, John E, Torres-Mejia G, Stern M, Lundgreen A, Hines L, Giuliano A, Baumgartner K, Herrick J and Wolff RK: Matrix metalloproteinase genes are associated with breast cancer risk and survival: The breast cancer health disparities study. PLoS One 8(5): e63165, 2013. PMID: 23696797. DOI: 10.1371/journal.pone.0063165

22 Néjima DB, Zarkouna YB, Gammoudi A, Manai M and Boussen H: Prognostic impact of polymorphism of matrix metalloproteinase-2 and metalloproteinase tissue inhibitor-2 promoters in breast cancer in tunisia: Case-control study. Tumour Biol 36(5): 3815-3822, 2015. PMID: 25656607. DOI: $10.1007 /$ s13277-014-3023-5

23 Habel AF, Ghali RM, Bouaziz H, Daldoul A, Hadj-Ahmed M, Mokrani A, Zaied S, Hechiche M, Rahal K and YacoubiLoueslati B: Common matrix metalloproteinase- 2 gene variants and altered susceptibility to breast cancer and associated features in tunisian women. Tumour Biol 41(4): 1010428319845749, 2019. PMID: 31014197 . DOI: $10.1177 / 1010428319845749$

24 Toth M, Sohail A and Fridman R: Assessment of gelatinases (mmp-2 and mmp-9) by gelatin zymography. In: Metastasis research protocols. Springer, $p p$. 121-135, 2012.

25 La Rocca G, Pucci-Minafra I, Marrazzo A, Taormina P and Minafra S: Zymographic detection and clinical correlations of mmp-2 and mmp-9 in breast cancer sera. Br J Cancer 90(7): 1414, 2004. PMID: 15054465. DOI: 10.1038/sj.bjc.6601725

26 Somiari SB, Shriver CD, Heckman C, Olsen C, Hu H, Jordan R, Arciero C, Russell S, Garguilo $G$ and Hooke J: Plasma concentration and activity of matrix metalloproteinase 2 and 9 in patients with breast disease, breast cancer and at risk of developing breast cancer. Cancer Lett 233(1): 98-107, 2006. PMID: 16473671. DOI: 10.1016/j.canlet.2005.03.003

27 Somiari SB, Somiari RI, Heckman CM, Olsen CH, Jordan RM, Russell SJ and Shriver CD: Circulating MMP2 and MMP9 in breast cancer-potential role in classification of patients into low risk, high risk, benign disease and breast cancer categories. Int J Cancer 119(6): 1403-1411, 2006. PMID: 16615109. DOI: 10.1002/ijc.21989

28 Katunina A, Gershtein E, Ermilova V, Tereshkina I, Nazarenko AY, Tyleuova A, Dvorova E, Karabekova Z, Gritskevich M and Berezov T: Matrix metalloproteinases 2, 7, and 9 in tumors and sera of patients with breast cancer. Bull Exp Biol Med 151(3): 359362, 2011. PMID: 22451887. DOI: 10.1007/s 10517-011-1330-z

29 Kim Y, Ollberding NJ, Shvetsov YB, Franke AA, Wilkens LR, Maskarinec G, Hernandez BY, Le Marchand L, Henderson BE 
and Kolonel LN: Plasma matrix metalloproteinases and postmenopausal breast cancer risk: A nested case-control study in the multiethnic cohort study. Breast Cancer Res Treat 136(3): 837-845, 2012. PMID: 23112106. DOI: 10.1007/s10549-0122308-x

30 Aroner SA, Rosner BA, Tamimi RM, Tworoger SS, Baur N, Joos TO and Hankinson SE: Plasma matrix metalloproteinase 2 levels and breast cancer risk. Cancer Epidemiol 39(3): 321-327, 2015. PMID: 25799912. DOI: 10.1016/j.canep.2015.02.010

31 Sheen-Chen SM, Chen HS, Eng HL, Sheen CC and Chen WJ: Serum levels of matrix metalloproteinase 2 in patients with breast cancer. Cancer Lett 173(1): 79-82, 2001. PMID: 11578812. DOI: $10.1016 / \mathrm{s} 0304-3835(01) 00657-7$

32 Patel S, Sumitra G, Koner B and Saxena A: Role of serum matrix metalloproteinase-2 and-9 to predict breast cancer progression. Clin Biochem 44(10-11): 869-872, 2011. PMID: 21565179. DOI: 10.1016/j.clinbiochem.2011.04.019

33 Shipley JM, Doyle GA, Fliszar CJ, Ye QZ, Johnson LL, Shapiro SD, Welgus HG and Senior RM: The structural basis for the elastolytic activity of the 92-kda and 72-kda gelatinases. Role of the fibronectin type ii-like repeats. J Biol Chem 271(8): 43354341, 1996. PMID: 8626782. DOI: 10.1074/jbc.271.8.4335

34 Visse R and Nagase H: Matrix metalloproteinases and tissue inhibitors of metalloproteinases: Structure, function, and biochemistry. Circ Res 92(8): 827-839, 2003. PMID: 12730128. DOI: 10.1161/01.RES.0000070112.80711.3D

35 Price SJ, Greaves DR and Watkins H: Identification of novel, functional genetic variants in the human matrix metalloproteinase-2 gene: Role of $\mathrm{Sp} 1$ in allele-specific transcriptional regulation. J Biol Chem 276(10): 7549-7558, 2001. PMID: 11114309. DOI: 10.1074/jbc.M010242200

36 Harendza S, Lovett DH, Panzer U, Lukacs Z, Kuhnl P and Stahl RA: Linked common polymorphisms in the gelatinase a promoter are associated with diminished transcriptional response to estrogen and genetic fitness. J Biol Chem 278(23): 2049020499, 2003. PMID: 12657623. DOI: 10.1074/jbc.M211536200

37 Yu C, Zhou Y, Miao X, Xiong P, Tan W and Lin D: Functional haplotypes in the promoter of matrix metalloproteinase-2 predict risk of the occurrence and metastasis of esophageal cancer. Cancer Res 64(20): 7622-7628, 2004. PMID: 15492291. DOI: 10.1158/0008-5472.CAN-04-1521

38 Yari K, Rahimi Z, Moradi MT and Rahimi Z: The MMP-2-735 $\mathrm{C}$ allele is a risk factor for susceptibility to breast cancer. Asian Pac J Cancer Prev 15(15): 6199-6203, 2014. PMID: 25124598. DOI: 10.7314/apjcp.2014.15.15.6199

39 Opdenakker G, Van den Steen PE and Van Damme J: Gelatinase B: A tuner and amplifier of immune functions. Trends Immunol 22(10): 571-579, 2001. PMID: 11574282. DOI: 10.1016/s14714906(01)02023-3

$40 \mathrm{Wu}$ ZS, Wu Q, Yang JH, Wang HQ, Ding XD, Yang F and Xu $\mathrm{XC}$ : Prognostic significance of MMP-9 and TIMP-1 serum and tissue expression in breast cancer. Int J Cancer 122(9): 20502056, 2008. PMID: 18172859. DOI: $10.1002 /$ ijc 23337

41 Zhang B, Ye S, Herrmann SM, Eriksson P, de Maat M, Evans A, Arveiler D, Luc G, Cambien F, Hamsten A, Watkins H and Henney AM: Functional polymorphism in the regulatory region of gelatinase $\mathrm{b}$ gene in relation to severity of coronary atherosclerosis. Circulation 99(14): 1788-1794, 1999. PMID: 10199873. DOI: 10.1161/01.cir.99.14.1788
42 Felizi RT, Veiga MG, Carelli Filho I, Souto RPD, Fernandes CE and Oliveira E: Association between matrix metallopeptidase 9 polymorphism and breast cancer risk. Rev Bras Ginecol Obstet 40(10): 620-624, 2018. PMID: 30352460. DOI: 10.1055/s-00381673366

43 Chiranjeevi P, Spurthi KM, Rani NS, Kumar GR, Aiyengar TM, Saraswati M, Srilatha G, Kumar GK, Sinha S and Kumari CS: Gelatinase B $(-1562 \mathrm{C} / \mathrm{T})$ polymorphism in tumor progression and invasion of breast cancer. Tumour Biol 35(2): 1351-1356, 2014. PMID: 24357512. DOI: 10.1007/s13277-013-1181-5

44 Padala C, Tupurani MA, Puranam K, Gantala S, Shyamala N, Kondapalli MS, Gundapaneni KK, Mudigonda S, Galimudi RK, Kupsal K, Nanchari SR, Chavan U, Chinta SK, Mukta S, Satti $\mathrm{V}$ and Hanumanth SR: Synergistic effect of collagenase-1 (MMP1), stromelysin-1 (MMP3) and gelatinase-b (MMP9) gene polymorphisms in breast cancer. PLoS One 12(9): e0184448, 2017. PMID: 28961241. DOI: 10.1371/journal.pone.0184448

45 Rahimi Z, Yari K and Rahimi Z: Matrix metalloproteinase-9-1562t allele and its combination with mmp-2-735 $\mathrm{c}$ allele are risk factors for breast cancer. Asian Pac J Cancer Prev 16(3): 1175-1179, 2015. PMID: 25735351. DOI: 10.7314/apjcp.2015.16.3.1175

46 Beeghly-Fadiel A, Lu W, Shu X-O, Long J, Cai Q, Xiang Y, Gao Y-T and Zheng W: Mmp9 polymorphisms and breast cancer risk: A report from the shanghai breast cancer genetics study. Breast Cancer Res Treat 126(2): 507-513, 2011. PMID: 20725776. DOI: $10.1007 / \mathrm{s} 10549-010-1119-1$

47 Resler AJ, Malone KE, Johnson LG, Malkki M, Petersdorf EW, McKnight B and Madeleine MM: Genetic variation in tlr or nfkappab pathways and the risk of breast cancer: A case-control study. BMC Cancer 13(1): 219, 2013. PMID: 23634849. DOI: 10.1186/1471-2407-13-219

48 Chahil JK, Munretnam K, Samsudin N, Lye SH, Hashim NAN, Ramzi NH, Velapasamy S, Wee LL and Alex L: Genetic polymorphisms associated with breast cancer in malaysian cohort. Indian J Clin Biochem 30(2): 134-139, 2015. PMID: 25883419. DOI: $10.1007 / \mathrm{s} 12291-013-0414-0$

49 Tesfaigzi Y, Myers OB, Stidley CA, Schwalm K, Picchi M, Crowell RE, Gilliland FD and Belinsky SA: Genotypes in matrix metalloproteinase 9 are a risk factor for copd. Int J Chron Obstruct Pulmon Dis 1(3): 267-278, 2006. PMID: 18046864 DOI: $10.2147 /$ copd.2006.1.3.267

50 Gerlach RF, Uzuelli JA, Souza-Tarla CD and Tanus-Santos JE: Effect of anticoagulantes on the determination of plasma matrix metalloproteinase (MMP)-2 and MMP-9 activities. Anal Biochem 34(1): 147-149, 2005. PMID: 15950912. DOI: 10.1016/j.ab.2005.04.038

51 Gerlach RF, Demacq C, Jung K and Tanus-Santos JE: Rapid separation of serum does not avoid artificially higher matrix metalloproteinase (MMP)-9 levels in serum versus plasma. Clin Biochem 40(1-2): 119-123, 2007. PMID: 17150202. DOI: 10.1016/j.clinbiochem.2006.10.007 